\title{
Left Small Bowel Syndrome A Case Report and Literature Review
}

Diarra Daouda $^{1 *}$, Salihou Abdoulfatihii ${ }^{1}$, Dzota Johane ${ }^{1}$, Laoudihy Dalale ${ }^{1}$, Chbani Kamilia ${ }^{1}$, SALAM Siham ${ }^{1}$, EL Ouzidane Lahcen ${ }^{1}$

${ }^{1}$ Pediatric Radiology Service Abderrahim Harouchi Children's Hospital, Ibn Rochd University Hospital Center of Casanblanca, Hassan II University of Casablanca, Morocco

DOI: $10.36347 /$ simcr.2022.v10i01.009

| Received: 27.11.2021 | Accepted: 01.01.2022 | Published: 29.01.2022

*Corresponding author: Diarra Daouda

Pediatric Radiology Service Abderrahim Harouchi Children's Hospital, Ibn Rochd University Hospital Center of Casanblanca, Hassan II University of Casablanca, Morocco

\section{Abstract}

Left small colon syndrome is a rare cause of neonatal obstruction, favored by the existence of a diabetic mother in 40 to $50 \%$ of cases. The diagnosis is evoked by ultrasound and confirmed by an opaque enema with water-solubles. The enema will allow a colonic emptying often accelerating the regression of the digestive symptoms (use of gastrografine if necessary). The prognosis is good with symptomatic and conservative treatment. The meconium plug syndrome can also be compared to this condition, which is also part of the functional occlusive syndromes that resolve.

Keywords: Left small colon - ASP - Enema opaque to water-soluble - Ultrasound.

Copyright $\odot 2022$ The Author(s): This is an open-access article distributed under the terms of the Creative Commons Attribution 4.0 International License (CC BY-NC 4.0) which permits unrestricted use, distribution, and reproduction in any medium for non-commercial use provided the original author and source are credited.

\section{INTRODUCTION}

The left small colon syndrome is a low neonatal occlusion where the opaque enema with watersoluble products has a doublé interest, diagnostic and therapeutic. It is favored by the existence of a diabetic mother in 40 to $50 \%$ of cases; the absence of elimination of meconium beyond 24 hours and a newborn with low birth weight [1]. The opaque enema with water-soluble is the diagnostic and therapeutic means. Our aim is to discuss this exceptional entity through our case and a review of the literature.

\section{Case Presentation}

A neonate at two days of life, at the end of a normal pregnancy with a birth weight of $3150 \mathrm{~g}$, was referred to the pediatric surgical emergency department at the 28th hour for a picture of neonatal obstruction (abdominal bloating, bilious vomiting with absence of meconium emission). The physical examination showed abdominal bloating with diffuse tympany, the progression of a rectal tube was normal.

An unprepared abdomen (UAP) showed moderate, diffuse bowel distension without hydroaeric levels, and the gastrografin water-soluble opaque enema showed complete opacification of the colon and reduction in the caliber of the rectum and individual colonic segments, with no disparity in caliber and no extravasation of product (Fig. $1 \mathrm{a}, \mathrm{b}$ and c).
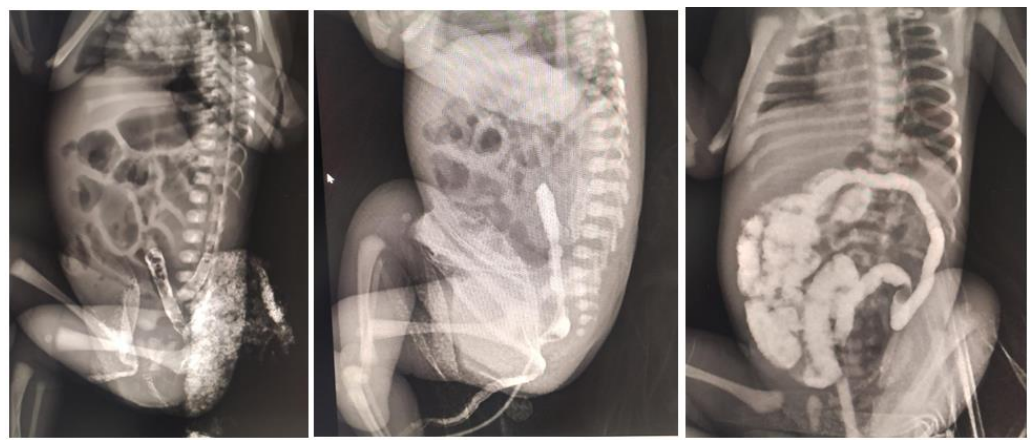

Fig-1: a, b and c: colonic enema with gastrografine: left small colon a: colonic distension without hydro-aeric level, b: beginning of filling, c: reduction of the caliber of the rectum and of the different colonic segments 


\section{DiSCUSSION}

Left small bowel syndrome is à very rare cause of neonatal low bowel obstruction of functional origin, rapidly resolving after a water-soluble enema [1]. Two theories of etiopathogeny have been put forward, the first is that of a disorder of colonic motility due to functional immaturity of the nerve plexuses and the second involves hormonal mechanisms, particularly in newborns of diabetic mothers where hypoglycemia increases vagal activity in the distribution zones of the vagus nerve which terminates at the left colonic angle. Thus, the typical radiological aspect of this syndrome is realized, which heals after correction of hypoglycemia and with the help of enema $[1,2]$.

The clinical context is that of a lower intestinal obstruction, with abdominal distension, delayed or no meconium emission and sometimes bilious vomiting. It usually occurs in the first three days of life, often in newborns with low birth weight or born to diabetic mothers (40 to $50 \%$ of cases) [3, 4]. The rectal probe test eliminates anal membrane, atresia or rectal stenosis [5].

The diagnosis can be evoked by an abdominal ultrasound which shows a left colon of small caliber, right colon and rectum of normal caliber. The unprepared abdomen shows nothing special apart from signs of lower intestinal obstruction and the appearance of the water-soluble opaque enema is very suggestive [6].

The evolution is spontaneously favorable with a regression of the symptomatology in a few hours after evacuation of the contrast product with meconium of variable aspect. The recovery of the digestive function is progressive. The elimination of Hirschsprung's disease and cystic fibrosis is mandatory [7].

\section{CONCLUSION}

Left small colon syndrome is an extremely rare cause of low neonatal obstruction of functional origin, occurring most often in premature babies or newborns of diabetic mothers where the opaque enema with water-soluble is the diagnostic and therapeutic means.

\section{REFERENCES}

1. Davis, W. S., Allen, R. P., Favara, B. E., \& Slovis, T. L. (1974). Neonatal small left colon syndrome. American Journal of Roentgenology, 120(2), 322-329.

2. Nixon, G. W., Condon, V. R., \& Stewart, D. R. (1975). Intestinal perforation as a complication of the neonatal small left colon syndrome. The American journal of roentgenology, radium therapy, and nuclear medicine, 125(1), 75-80.

3. Stewart, D. R., Nixon, G. W., Johnson, D. G., \& Condon, V. R. (1977). Neonatal small left colon syndrome. Annals of surgery, 186(6), 741.

4. Philippart, A. I., Reed, J. O., \& Georgeson, K. E. (1975). Neonatal small left colon syndrome: intramural not intraluminal obstruction. Journal of pediatric surgery, 10(5), 733-740.

5. Amrani, A., Zerhouni, H., Hachimi, M. H., Ettayebi, F., \& Benhammou, M. (2002). Le syndrome du «petit côlon gauche»(à propos de deux cas). Archives de pédiatrie, 9(9), 917-920.

6. Daudet, M. (1979). Les faux hirschsprung dans les occlusions neonatales: a propos de 6 observations (1 meconium plus syndrome, 2 immaturites des plexus myenteriques, 1 petit colon gauche et 2 cas familiaux d'intestin adynamique chronique).

7. Heaton, N. D., Howard, E. R., \& Garrett, J. R. (1991). Small left colon syndrome: an immature enteric plexus. Journal of the Royal Society of Medicine, 84(2), 113-114. 